\title{
Fasudil attenuates soluble fms-like tyrosine kinase-1 (sFlt-1)- induced hypertension in pregnant mice through RhoA/ROCK pathway
}

\author{
Ying Gu${ }^{1}$, Yaling Feng ${ }^{1}$, Jinjin Yư ${ }^{2}$, Hua Yuan ${ }^{1}$, Yongxiang Yin ${ }^{3}$, Jian Ding ${ }^{1}$, Jun \\ $Z{ }^{1}$, Yaohui $X u^{1}$, Jianjuan $X u^{1}$ and Haisha $C^{1}$ \\ ${ }^{1}$ Department of Obstetrics and Gynecology, Wuxi Maternal and Child Health Hospital Affiliated to Nanjing Medical University, \\ Wuxi, Jiangsu 214002, PR China \\ ${ }^{2}$ Department of Obstetrics and Gynecology, The Affiliated Hospital of Jiangnan University (Wuxi Fourth People's Hospital), \\ Wuxi, Jiangsu 214062, PR China \\ ${ }^{3}$ Department of Pathology, The Affiliated Maternity and Child Health Hospital of Nanjing Medical University, Wuxi, Jiangsu \\ 214002, PR China \\ Correspondence to: Yaling Feng, email: 15161506909@163.com \\ Jinjin Yu, email: fengyl523@sina.com \\ Keywords: preeclampsia; fasudil; endothelial dysfunction; hypertension; RhoA/ROCK \\ Received: July 22, $2017 \quad$ Accepted: September 23, $2017 \quad$ Published: October 24, 2017 \\ Copyright: $\mathrm{Gu}$ et al. This is an open-access article distributed under the terms of the Creative Commons Attribution License \\ 3.0 (CC BY 3.0), which permits unrestricted use, distribution, and reproduction in any medium, provided the original author and \\ source are credited.
}

\section{ABSTRACT}

Preeclampsia (PE) has become the leading cause of maternal and fetal morbidity and mortality in the world, which is characterized by a systemic maternal inflammatory response associated with endothelial dysfunction, hypertension, and proteinuria. The development of PE is still barely predictable and thus challenging to prevent and manage clinically. Fasudil (FSD), the first-generation Rho/ROCK inhibitor, has been studied widely and applied in clinical practice with high safety and efficacy in treating hypertension and other cardiovascular diseases. However, few studies have focused on the effect of fasudil on preeclampsia in vivo and in vitro. Therefore, the aim of this study is to investigate the effects of fasudil on hypoxia/reoxygenation injury in vitro and its role on preeclamptic animal model. Here, we found that RhoA/ROCK pathway was significantly activated in $\mathbf{H} / \mathbf{R}$-challenged endothelial cells and in placenta and umbilical vessel of PE mice. And fasudil pre-treatment can protect vascular endothelial cells from H/R-induced apoptosis. In addition, inhibition of RhOA/ROCK pathway with fasudil can reduce the high blood pressure and urine protein levels as well as the concentration of s-Flt in peripheral and umbilical blood in a dose-dependent manner, thus resulting in prevention of the development of PE. Thus, Fasudil attenuates soluble fms-like tyrosine kinase-1 (sFlt-1)-induced hypertension in pregnant mice through RhoA/ROCK pathway, which would become a potential strategy for PE therapy.

\section{INTRODUCTION}

Preeclampsia has become the leading cause of maternal and fetal morbidity and mortality in the world accounting for nearly $40 \%$ of births delivered at early gestation, which is characterized by a systemic maternal inflammatory response associated with endothelial dysfunction, hypertension, and proteinuria [1]. There is also evidence that the risk of subsequent cardiovascular disease is significantly increased in women affected by preeclamptic pregnancies [2]. Although the development of PE is still barely predictable and thus challenging to prevent and manage clinically, there is evidence that placental oxidative stress attributable to abnormal uteroplacental blood circulation plays a critical role [3].

Oxidative stress of the placenta is able to result in endothelial dysfunction. Several important antioxidants, such as superoxide dismutase (SOD) and glutathione 
peroxidase (GPx), are significantly decreased in the maternal circulation of women with preeclampsia [4]. A simple in vitro model wherein endothelial cell monolayers are exposed to hypoxia/reoxygenation $(\mathrm{H} / \mathrm{R})$ is widely used to mimic the microvascular dysfunction that is induced by oxidative stress [5]. And administration of antioxidant vitamins $\mathrm{C}$ and $\mathrm{E}$ can block H/R-mediated sFlt-1 secretion involving in inhibition of p38 signaling, and increase the secretions of sFlt- 1 have been shown to participate in the pathogenesis of pre-eclampsia $[6,7]$. Our previous study has demonstrated that exogenous alpha-1 antitrypsin alleviated hypoxia/reoxygenation injury in a dose- and time- dependent manner via inactivation of Rac1/p38 signaling and suppression of oxidative stress [8]. And exogenous alpha-1 antitrypsin injection increases the antioxidants and suppresses oxidative stress, and subsequent prevention of PE development [9]. Thus, antioxidant treatment may be a potential strategy for preeclampsia therapy.

It was reported that $\mathrm{H} / \mathrm{R}$-induced changes in endothelial permeability result from coordinated actions of the Rho GTPases Rac1 and RhoA, suggesting that Rho GTPases act as key mediators coupling cellular redox state to endothelial function [10]. Ras homolog gene family, member A (RhoA) was expressed in syncytiotrophoblasts and cytotrophoblasts. The results of immunochemistry staining and qPCR showed that RhoA protein and mRNA expression in placental tissues of mild and severe preeclampsia groups was significantly higher than that in normal pregnancy, indicating that increased expression
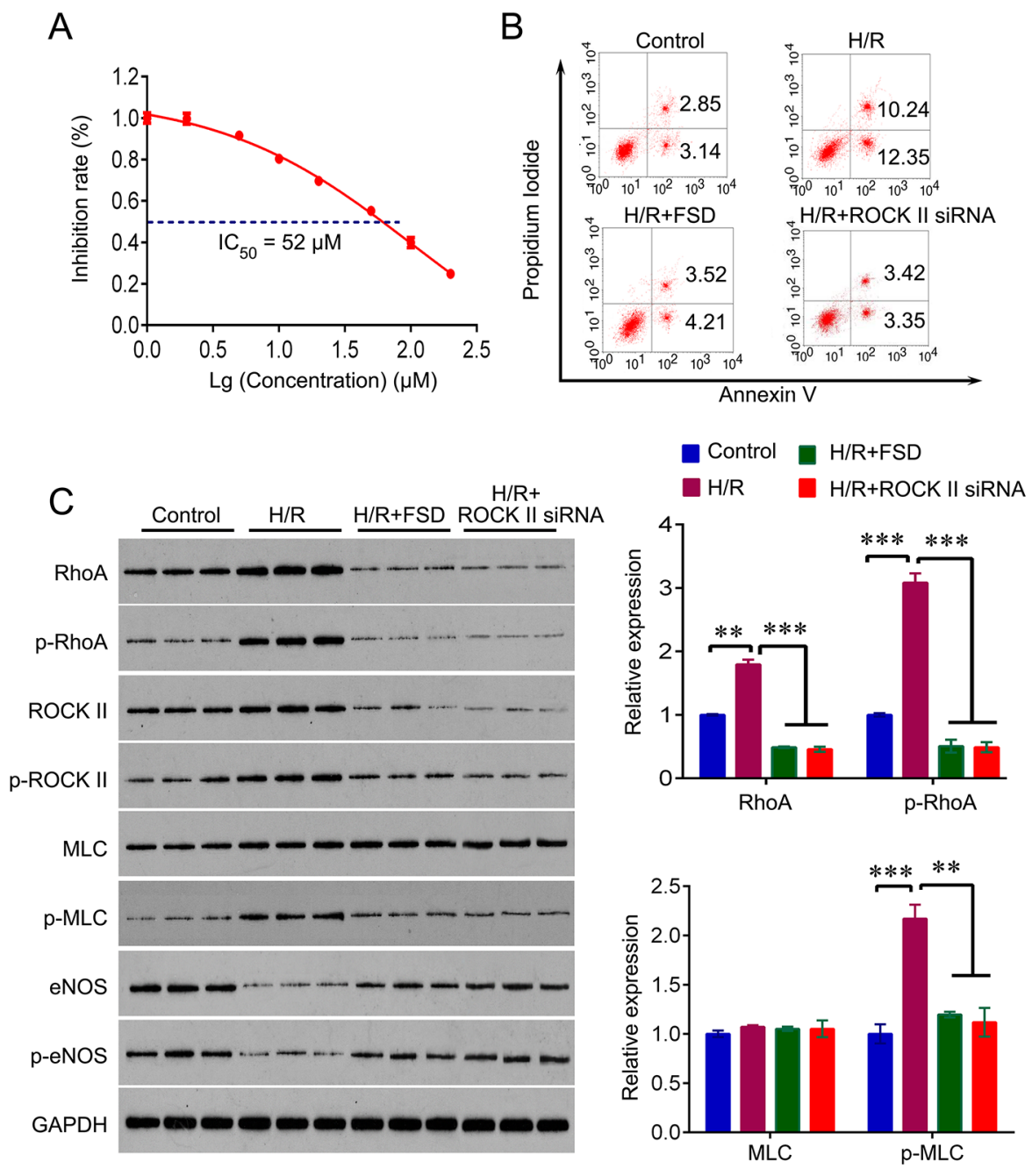
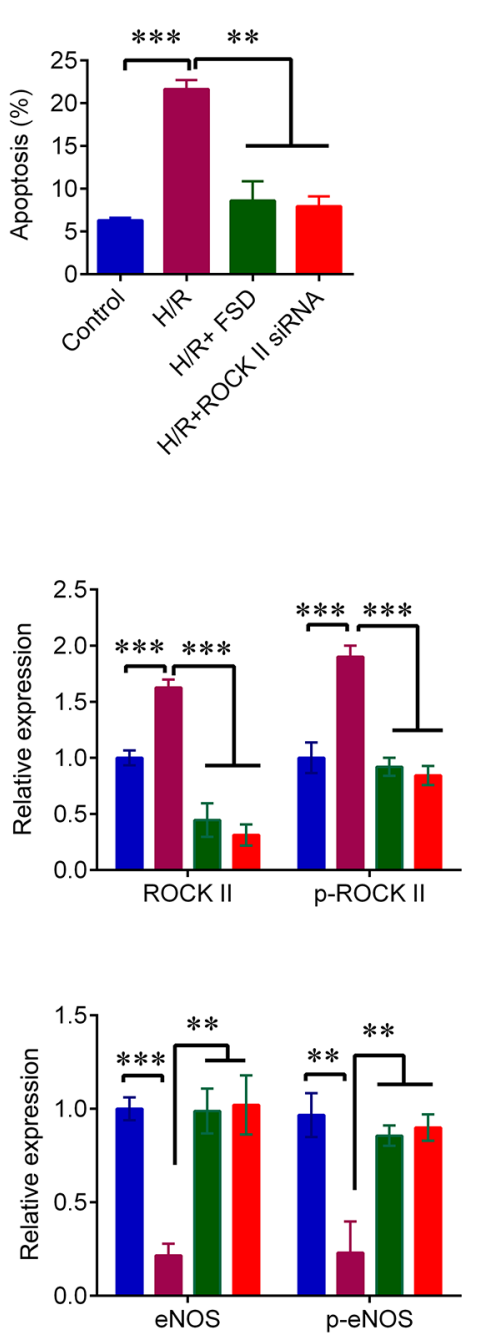

Figure 1: Fasudil protects HUVEC cells from H/R-induced apoptosis. (A) MTT was used to determine the IC ${ }_{50}$ of FSD in HUVEC cells cultured in standard condition. (B) Flow cytometry was used to measure the apoptosis rate in HUVEC cells with indicated treatment. HUVEC cells cultured in standard condition were used as control. (C) Western blot analysis for RhoA, ROCK, MLC, eNOS and their phosphorylated form in HUVEC cells with indicated treatment. The experiments were independently repeated for three times. The experiments were independently repeated three times. Data were expressed as mean \pm standard error. ANOVA with post hoc Tukey's test was used for statistical analyses. H/R, Hypoxia-reoxygenation; FSD, fasudil. ${ }^{*} \mathrm{p}<0.05,{ }^{* *} \mathrm{p}<0.01,{ }^{* * *} \mathrm{p}<0.001$. 
of RhoA in placental tissues might play an important role in the pathogenesis of preeclampsia [11]. During preeclampsia, the release of reactive oxygen species might activate the RhoA kinase pathway to enhance vascular reactivity. Pretreatment with superoxide dismutase/ catalase to quench reactive oxygen species or RhoA kinase inhibitor blocked enhanced responses in preeclamptic and normal vessels [12]. Fasudil (FSD), the first-generation Rho/ Rho-associated protein kinase (ROCK) inhibitor, has been studied widely and applied in clinical practice with high safety and efficacy in treating hypertension and other cardiovascular diseases [13]. Butruille L et al showed that fasudil exposure during late gestation alleviates the growth of intrauterine growth-restricted fetuses from hypertensive rat mothers [14]. However, few studies have focused on the effect of fasudil on preeclampsia in vivo and in vitro. Therefore, the aim of this study is to investigate the effect of fasudil on hypoxia/reoxygenation injury in vitro and its role on preeclamptic animal model.

\section{RESULTS}

\section{Fasudil protects HUVEC cells from hypoxia/ reoxygenation induced apoptosis}

We firstly determined the $\mathrm{IC}_{50}$ of Fasudil (FSD) in HUVEC cells. HUVEC cells were treated with a range of concentrations of Fasudil for $8 \mathrm{hr}$, and MTT were used to measure the cell viability. As shown in Figure 1A, the cell viability of HUVEC was decreased with increasing of concentrations of Fasudil; and the $\mathrm{IC}_{50}$ was determined at $52 \mu \mathrm{M}$.

We then used a well-established model of hypoxia/ reoxygenation $(\mathrm{H} / \mathrm{R})$ treatment that causes vascular endothelial cells injury in HUVEC cells to investigate the role of Fasudil in H/R injury. HUVEC cells underwent 4 $\mathrm{hr}$ of hypoxia followed by $18 \mathrm{hr}$ of reoxygenation. We pretreated HUVEC cells with $50 \mu \mathrm{M}$ Fasudil for $8 \mathrm{hr}$ before $\mathrm{H} / \mathrm{R}$ challenge. And the cells with no treatment were used as control. Exposure of HUVEC cells to H/R led to a significant increase in cell apoptosis $(22.5 \%)$, while Fasudil pre-treatment significantly decreased percentage of apoptosis (7.7\%) undergoing $\mathrm{H} / \mathrm{R}$ challenge (Figure 1B), suggesting a protective effect of Fasudil against $H / R$ injury. We further confirmed that inhibition of ROCK II by using siRNA markedly reduced the apoptosis induced by $H / R$ (Figure 1B). In addition, we also analyzed the expression of key molecules of RhoA signaling, including RhoA, ROCK II, MLC and eNOS. We found that H/R treatment significantly upregulated RhoA and ROCK II expression and their phosphorylated form, and increased the expression of phosphorylated MLC, whereas reduced the expression of eNOS and p-eNOS. However, Fasudil pre-treatment and ROCK II knockdown reversed H/Rmediated effects on these molecules (Figure 1C), indicating that protective effect of Fasudil against $\mathrm{H} / \mathrm{R}$ injury is involved in RhoA signaling.

\section{Fasudil attenuates the hypertension induced by sFlt-1 in pregnant mice}

The results showed that sFlt-1 infusion significantly increased blood pressure on day 13 of gestation (Figure $2 \mathrm{~A}$ ), and elevated the urine protein levels compared with the normal group (Figure 2D), indicating that the PE model was successfully established. To investigate the role of Fasudil on PE, we injected Fasudil solution (10 or $50 \mathrm{mg} / \mathrm{kg}$ ) into tail veins every day from days 13 to 19 of gestation in PE mice, and $100 \mu \mathrm{l}$ of saline injection was used as a control. We found that Fasudil injection significantly relieved the high blood pressure, and reduced urine protein levels in a dose-dependent manner (Figure $2 \mathrm{~B}$ and 2D). In addition, we measured the circulating ACE expression using ELISA kit, and found that ACE level was significantly increased in PE mice compared with normal mice, and inhibiting ROCK with fasudil or with ROCK II siRNA can reduce circulating ACE expression (Figure 2C). We also observed the fact that the concentrations of s-Flt in serum from both peripheral blood and umbilical cord blood were significantly increased in PE mice (Figure 2E and 2F). As expected, the concentrations of s-Flt in serum from both peripheral blood and umbilical cord blood were reduced with increased dosage of Fasudil (Figure 2E and 2F). In addition, knockdown of ROCK II by siRNA significantly relieved the high blood pressure, and reduced urine protein levels as well as the concentrations of s-Flt in serum from both peripheral blood and umbilical cord blood (Figure 2). Thus, these results suggest that Fasudil injection is able to relieve the symptoms of PE mediated by sFlt infusion, which may be through inactivating ROCK pathway.

\section{Fasudil inactivates RhoA signaling that induced by $\mathrm{PE}$}

In addition, we obtained the placenta tissues from the pregnant mice to analyze Fasudil related downstream molecules. We found that PE activated the RhoA/ROCK signaling that enhanced the levels of these genes, including RhoA, ROCK II, p-MLC, as well as VEGF, but attenuated the levels of eNOS in both placenta and umbilical vessel tissues. We demonstrated that Fasudil was able to reduce the levels of RhoA, ROCK II, as well as VEGF, and the phosphorylation levels of MLC, but upregulated the levels of eNOS (Figure 3). Furthermore, we performed IHC assay to confirm that Fasudil injection significantly increased the expression of eNOS, and decreased the levels of RhoA, ROCK II and VEGF, as well as the phosphorylation levels of MLC that upregulated by PE in placenta tissues (Figure 3). And knockdown of ROCK 
II exerted the similar effects with Fasudil (Figures 3 and 4), indicating that prevention of the development of preeclampsia by Fasudil is involved in RhoA signaling.

\section{DISCUSSION}

In this study, we at first time demonstrate that fasudil pre-treatment can protect vascular endothelial cells from H/R-induced apoptosis, and fasudil injection is able to relieve the high blood pressure and reduce urine protein levels in a dose-dependent manner, and thus prevent the development of PE.

$\mathrm{H} / \mathrm{R}$, similar to ischemia-reperfusion, triggers cells damage due to augmented oxidative stress, mitochondrial dysfunction and inflammation [15]. A deleterious role of activated RhoA/ROCK signaling in ischemia-reperfusion injury has been demonstrated in several in vivo models [16-18]. ROCK inhibition with fasudil achieved less inflammation, reduced oxidative stress and lower apoptosis, which may be involved in activation of the $\mathrm{PI} 3 \mathrm{~K} / \mathrm{Akt} / \mathrm{eNOS}$ signaling pathway, preservation of endothelial function and suppression of inflammatory responses [13, 19, 20]. In line with previous studies, we also found that fasudil pre-treatment can decrease H/Rinduced apoptosis in vascular endothelial cells, which may through inhibition of RhoA/ROCK signaling and activation of eNOS signaling pathway [21].

Hypertension and oxidative stress are two hallmarkers of preeclampsia. Increased activity of RhoA/ ROCK pathway has been observed in experimental hypertension models and hypertensive patients, which appear to be the consequence of the up-regulation of

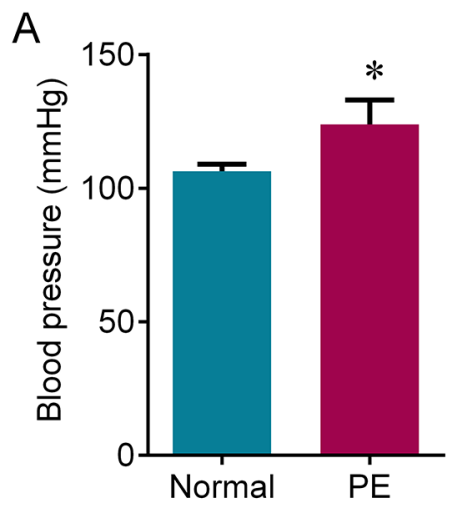

D

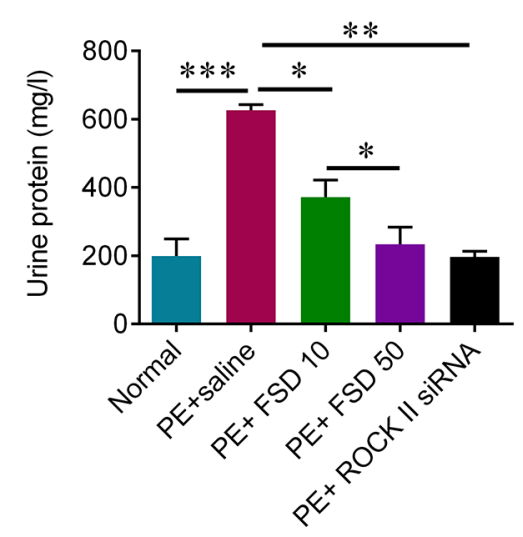

B

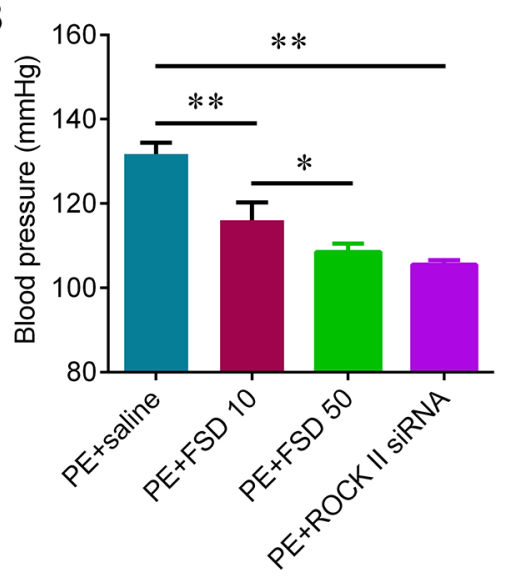

E

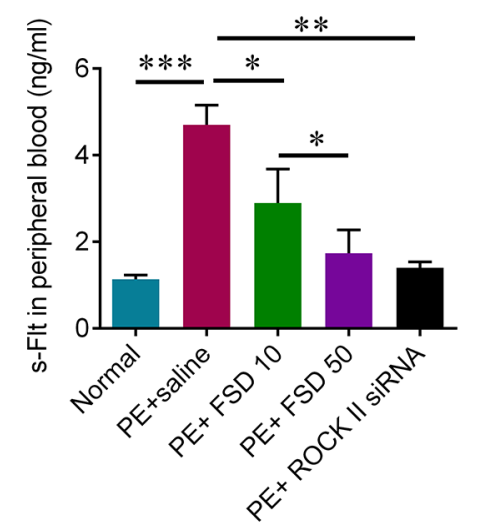

C

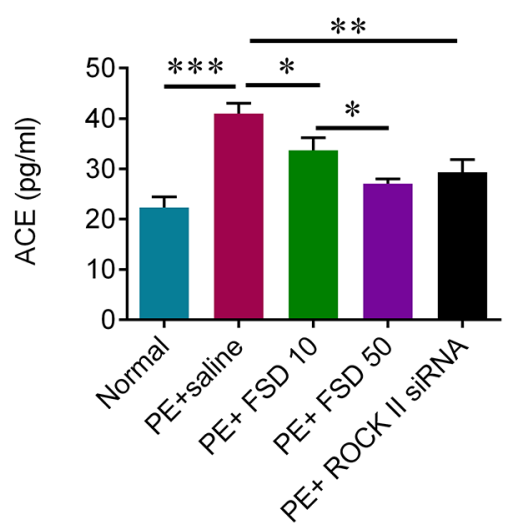

F

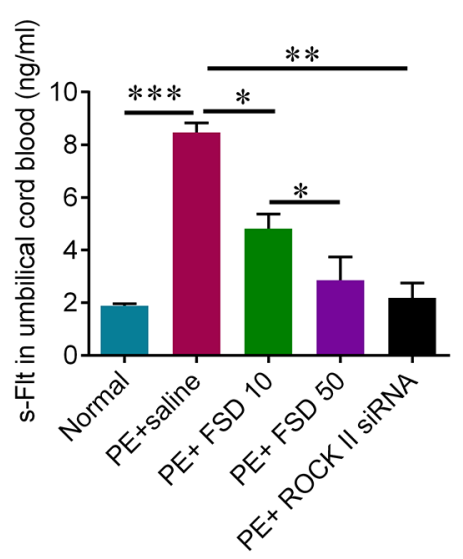

Figure 2: Fasudil decreases blood pressure induced by sFlt infusion in pregnant mice. (A) Non-invasive rat tail blood pressure detecting method was used to measure blood pressure on day 13 of gestation. Student t test was used for statistical analyses. (B) Non-invasive rat tail blood pressure detecting method was used to measure systolic blood pressure on day 19 of gestation after 6 days Fasudil treatment or ROCK knockdown by siRNA. (C) ELISA was used to detect the concentration of Angiotensin converting enzyme (ACE) in peripheral blood. (D) The proteinuria was measured on day 19 of gestation after 6 days Fasudil treatment or ROCK knockdown by siRNA. The PE mice treated with saline were used as control. ELISA was used to detect the concentration of s-Flt in peripheral blood (E) and cord blood (F). Number of animal for each group=8. Data were expressed as mean \pm standard error. ANOVA with post hoc Tukey's test was used for statistical analyses. ${ }^{*} \mathrm{p}<0.05,{ }^{* *} \mathrm{p}<0.01,{ }^{* * *} \mathrm{p}<0.001$. 
renin-angiotensin-aldosterone system and the increased production of reactive oxygen species (ROS) [22, 23]. ROCK, including two members ROCK1 and ROCK2, is one of the best-characterized effectors of small GTPase RhoA, which is involved in a wide range of fundamental cellular functions such as contraction, adhesion, migration, proliferation, and apoptosis [24, 25]. And ROCK is best known for promoting actin filament stabilization and generation of actin-myosin contractility by phosphorylating numerous downstream target proteins, including the myosin binding subunit of myosin light chain (MLC) [26-28]. Emerging evidence has demonstrated

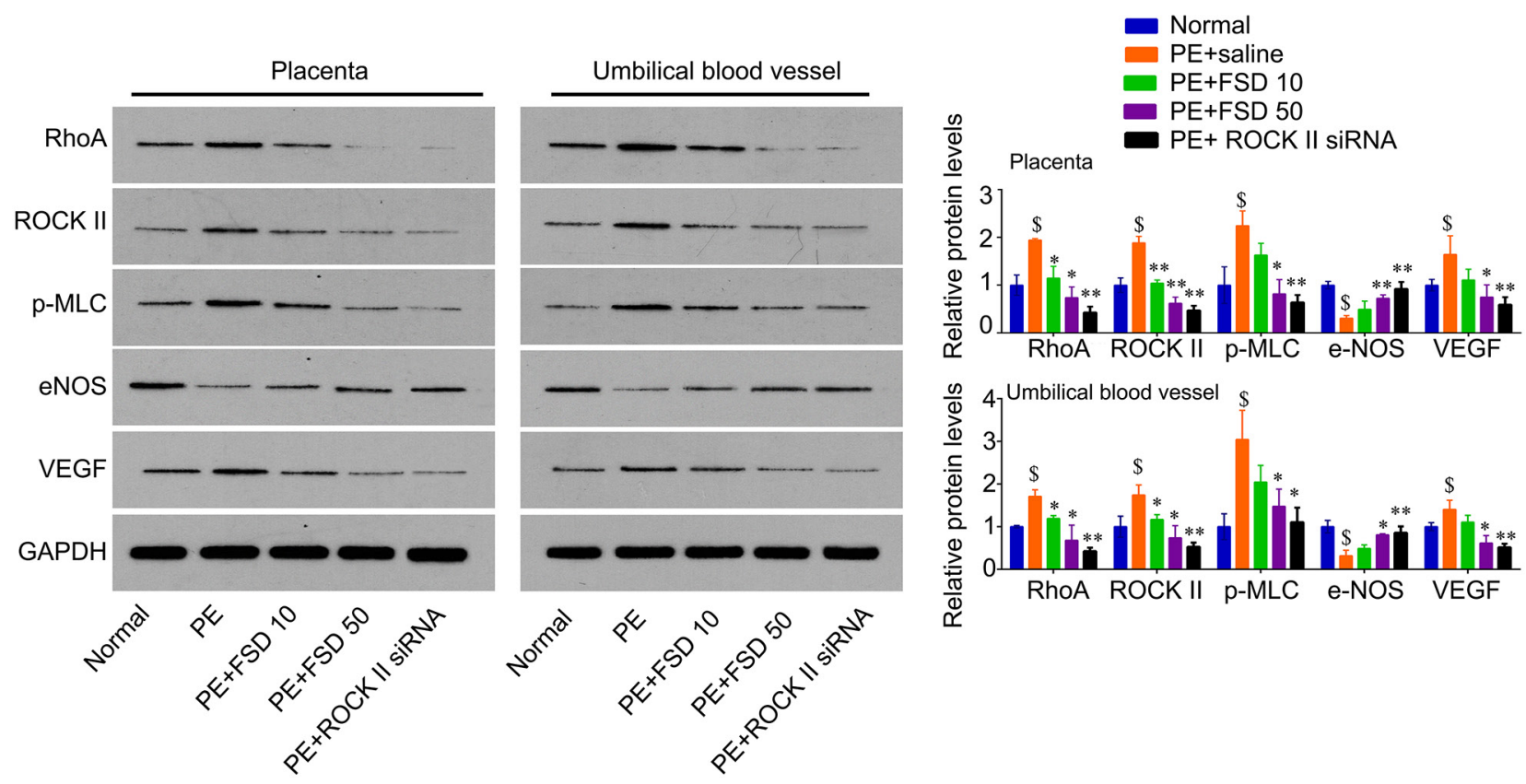

Figure 3: Fasudil regulates the expression of RhoA, ROCK, p-MLC, eNOS and VEGF in placenta and umbilical vessel from PE mice. Western blot analysis for RhoA, ROCK, p-MLC, eNOS and VEGF in mice placenta and umbilical vessel, and quantification. Number of animal for each group $=8$. Data were expressed as mean \pm standard error. ANOVA with post hoc Tukey's test was used for statistical analyses. $\$ \mathrm{p}<0.05$ vs. Normal. ${ }^{*} \mathrm{p}<0.05,{ }^{* *} \mathrm{p}<0.01$ vs. PE + saline group.
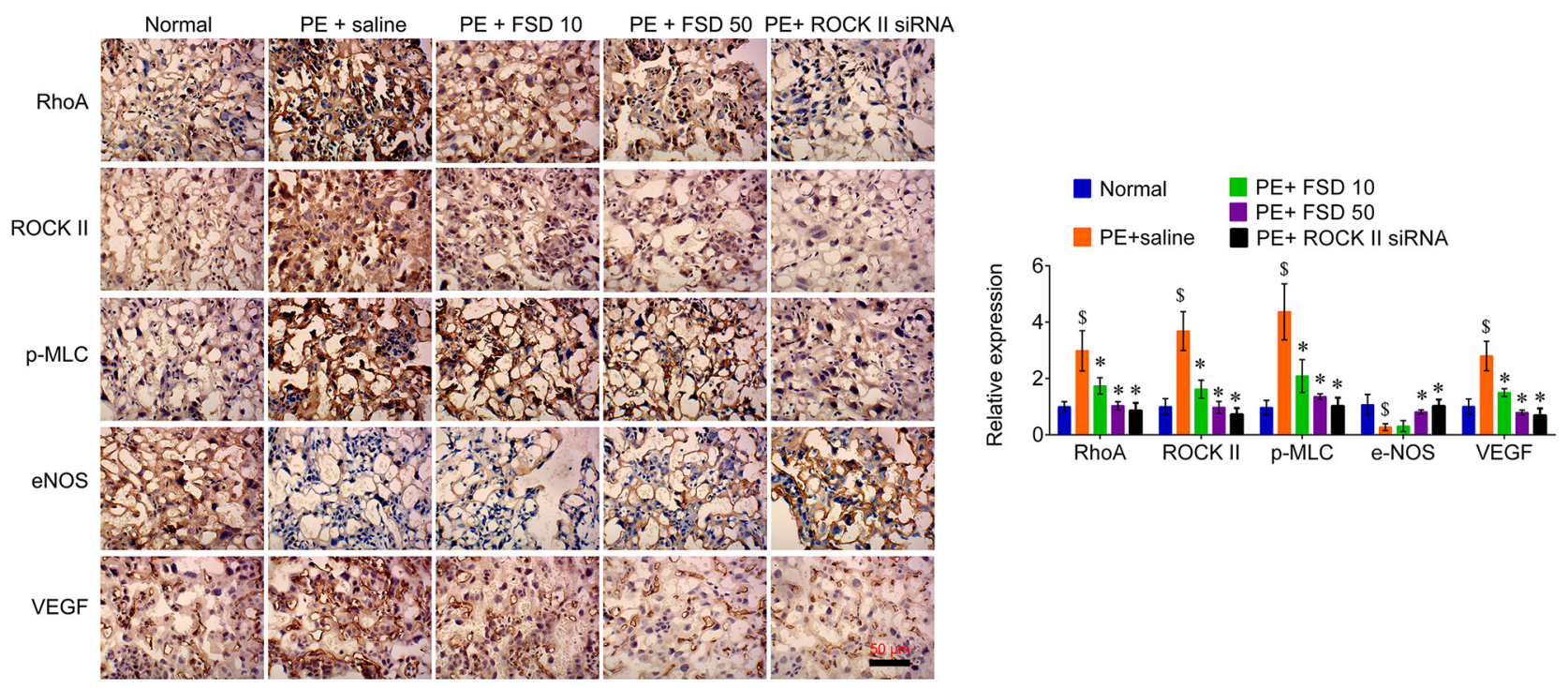

Figure 4: IHC analyzes the expression of RhoA, ROCK, p-MLC, eNOS and VEGF in placenta from PE mice. Representative images of IHC staining for RhoA, ROCK, p-MLC, eNOS and VEGF (left), and quantification (right). Bar=50 $\mu$; magnification, 200 $\times$. Number of animal for each group $=8$. Data were expressed as mean \pm standard error. ANOVA with post hoc Tukey's test was used for statistical analyses. $\$ \mathrm{p}<0.05$ vs. Normal. ${ }^{*} \mathrm{p}<0.05,{ }^{* *} \mathrm{p}<0.01$ vs. PE+saline group. 
the potential therapeutic application of ROCK inhibitors in cardiovascular diseases with a remarkable efficacy in reducing vascular smooth muscle cell hypercontraction, endothelial dysfunction, inflammatory cell recruitment and vascular remodeling [29].

Genetic studies also support the importance of the RhoA/ROCK pathway in blood pressure [30, 31]. ROCK inhibition by fasudil reduces smooth muscle contractility through decreasing MLC phosphorylation and improving endothelial function via restoring eNOS expression/ activity and NO production [21]. ROCK inactivation also reduces inflammation and remodeling through suppressing the expression of pro-inflammatory cytokines in endothelial and inhibiting reactive oxygen species (ROS) production [32, 33]. Increased blood pressure in preeclampsia is associated with marked vascular inflammation and ROS that may enhance vascular reactivity via the activation of RhoA/ROCK pathway [12]. Fasudil exposure during late gestation alleviates the growth of intrauterine growth restriction (IUGR) fetuses from hypertensive rat mothers, which may result from an improvement of maternal blood pressure induced by Fasudil [14]. Lopez NC et al found that fasudil decreases the function of the RhoA/ROCK pathway, reducing the pulmonary arterial pressure and resistance in chronically hypoxic highland neonatal lambs [34]. Here, we found that RhoA/ROCK pathway was significantly activated in placenta and umbilical vessel of PE mice. And inhibition of RhoA/ROCK pathway with fasudil can reduce the high blood pressure and urine protein levels as well as the concentration of s-Flt in peripheral and umbilical blood in a dose-dependent manner, thus resulting in prevention of the development of PE. However, more studies are needed to evaluate the long term effects of Fasudil treatment on maternal and newborn.

Summary, we here demonstrate that inhibition of ROCK with fasudil can protect vascular endothelial cells from H/R-induced apoptosis and prevent the development of PE. Thus, fasudil would become a potential strategy for PE therapy.

\section{MATERIALS AND METHODS}

\section{Cell culture and treatment}

Human Umbilical Vein Endothelial cells (HUVEC) were obtained from ATCC (American Type Culture Collection, Manassas, VA). The cells were cultured in DMEM supplemented with fetal bovine serum to a final concentration of $10 \%$ at $37^{\circ} \mathrm{C}$ in a humidified incubator containing $5 \% \mathrm{CO} 2$. The cells within 5 passages were used for experiments.

To knock down the level of ROCK II, we transfected ROCK II small interfering RNA (siRNA) into HUVEC cells (RiboBio Co., Ltd, Guangzhou, China) by using Lipofectamine 3000 (Life technologies, Grand Island, NY) in accordance with the manufacture's instruction. After 48 $\mathrm{h}$ transfection, the cells were used for further analysis.

\section{Hypoxia/reoxygenation model}

The hypoxia/reoxygenation model in HUVEC cells was established as we previously described [8]. For hypoxia, the cells were cultured in glucose and serum free media in normal incubator for 30 minutes, the cells then were exposed to hypoxia $\left(1 \% \mathrm{O}_{2}\right)$ in an AW200SG hypoxic workstation (ELECTROTEK, UK) using a continuous flow of a humidified mixture of $1 \% \mathrm{O}_{2}, 5 \%$ $\mathrm{CO}_{2}$, and $94 \% \mathrm{~N}_{2}$ at $37^{\circ} \mathrm{C}$ for $4 \mathrm{~h}$. For reoxygenation, after hypoxia, the cells were returned to culture in normal incubator with $20 \% \mathrm{O}_{2}, 5 \% \mathrm{CO}_{2}$, and $75 \% \mathrm{~N}_{2}$ at $37^{\circ} \mathrm{C}$ for $18 \mathrm{~h}$.

\section{Determination of $\mathrm{IC}_{50}$ of fasudil in HUVEC cells}

To determine the $\mathrm{IC}_{50}$ of Fasudil, the cells were treated with different concentrations of Fasudil for $8 \mathrm{~h}$, at which time cell proliferation rate were assessed by using MTT. Increasing Fasudil concentrations were designed as 2, 5, 10, 20, 50, 100 and $200 \mu \mathrm{mol}$.

\section{Flow cytometric analysis of apoptosis}

Cells from each group were trypsinizated and washed with cold PBS, and then stained by Annexin V-FITC/PI Apoptosis Detection Kit (BD Biosciences) according to the manufacturer's instructions. The cell apoptosis was analyzed by flow cytometry (Beckman Coulter, USA). The experiments were independently performed in triplicate.

\section{Preeclampsia-like model preparation}

The female C57/BL6N mice were obtained from Hunan SJA Co. (Changsha, China). Animals were housed in a temperature-controlled room $\left(23^{\circ} \mathrm{C}\right)$ with a 12:12 light: dark cycle. The experimental animals in this study were approved by the Institutional Animal Care and Research Advisory Committee of Nanjing Medical University. The preeclampsia model was prepared as we previously described. sFlt-1 (Recombinant mouse VEGF R1/Flt-1 Fc Chimera, Cat. No. 7756-FL-050, R\&D Systems) was infused at a rate of $3.7 \mu \mathrm{g} / \mathrm{kg} /$ day for 6 days (in sterile saline) beginning on day 7 of gestation via miniosmotic pump (model 2001; Alzet Scientific Corporation, Palto Alto, CA) into pregnant rats. Normal pregnant/control groups $(n=8)$ were fitted with a vehicle filled mini-osmotic pump. The preeclampsia mice were randomly divided into three groups each for 8 animals: $\mathrm{PE}+$ saline, PE+ FSD 10, PE+ FSD 50 and PE+ROCK siRNA. Lentivirus ROCK II siRNA $\left(2 \times 10^{9}\right.$ titer units (TU)/ml, GeneChem Corporation, Shanghai, China) were used to knock down the expression of ROCK II in PE 
mice. FSD solution ( 10 or $50 \mathrm{mg} / \mathrm{kg} /$ day for 6 days starting on day 13 of gestation, $n=8$ ) and lentiviral suspension (100 $\mu \mathrm{l} /$ day for 6 days starting on day 13 of gestation, $\mathrm{n}=8$ ) were injected into tail veins. The preeclampsia mice treated with saline $(n=8)$ were used as control.

\section{Blood pressure measurement and tissues collection}

Non-invasive tail-cuff Systolic BP measurements were carried out in conscious mice using the non-invasive automated sphygmomanometer (BP-98A, Softron, Beijing, China). The mice were placed in a 38 degree incubator and warmed for 15 minutes to stabilize the mood and to expand the local vessels sufficiently, and then the pressurized sensor was placed in the tail. Mice blood pressure was measured after a few seconds of sobriety and tranquility. Each mouse was continuously measured 5 times and the average was taken as the final result. Systolic BP measurements were performed at the same time to ensure that blood pressure is relatively stable.

The proteinuria and sFlt were measured on day 19. On day 19 of pregnancy, the animals were euthanized under the anesthesia by overdose chloral hydrate. The peripheral and cord blood were collected for further analysis. And placentas and umbilical vessel were collected for western blot and/or IHC analysis.

\section{Reagents}

Primary antibodies: rabbit monoclonal anti-RhoA (cat no. ab187027), rabbit monoclonal anti-ROCK II (cat no. ab71598), rabbit monoclonal anti-ROCK II (phospho Y722) (cat no. ab182648), rabbit monoclonal anti-eNOS (cat no. ab66127), rabbit monoclonal anti-eNOS (phospho S1177) (cat no. ab75639) from Abcam (Cambridge, UK); rabbit polyclonal anti-RhoA (Ser188) (cat no. bs-5330R), rabbit polyclonal anti-MLC (cat no. bs-7115R), rabbit polyclonal anti-phosphorylated MLC (cat no. bs-4060R) and rabbit polyclonal anti-VEGF (cat no. bs-1665R) from Bioss (Beijing, China); mouse monoclonal antiGAPDH (cat no. SC-365062) were from Santa Cruze (Dallas, Texas, USA). Horseradish peroxidase-conjugated secondary goat anti-mouse and goat anti-rabbit antibodies were from Boster (Wuhan, China). HRP-polymer antimouse IHC kit and HRP-polymer anti-rabbit IHC kit were from Maixin (Fuzhou, China). ELISA Kit for soluble fms-like tyrosine kinase-1 (sFlt-1) was from Cloud-Clone Corp. (cat no. SEB818Mu, Houston, TX, USA). Ace (Mouse) ELISA Kit for Angiotensin converting enzyme was from Abnova (cat no. KA3365, Taipei City, Taiwan).

\section{Immunohistochemical (IHC) staining}

The placenta tissues were embedded in paraffin and cut into $4 \mu \mathrm{m}$ slides. The slides were routinely deparaffinized and hydrated. After inactivating endogenous peroxidase in 3\% hydrogen peroxide, slides were then retrieved in citric acid buffer ( $\mathrm{pH6.0)}$ by microwave for 15 $\mathrm{min}$. Slices were blocked in normal goat serum for $30 \mathrm{~min}$ at room temperature, and incubated with primary antibody overnight at $4^{\circ} \mathrm{C}$. The slides were then washed with TBST and incubated with appropriate secondary antibody for 2 $\mathrm{h}$ at $37^{\circ} \mathrm{C}$. The sections were then washed with TBST and stained by using DAB Detection Kit (Solarbio, Beijing, China). Finally, the sections were counterstained with hematoxylin. The slices were captured and analyzed using Motic Images Advanced 3.2 software (Motic, XiaMen, China). The average optical density analysis was conducted by a researcher blind to treatment conditions. Data was acquired from eight sections/animal and the data were averaged to produce the relative expression of proteins.

\section{Western blot analysis}

RIPA lysis buffer was used to extract protein from indicated cells. BCA Protein Assay Kit (Thermo Scientific, USA) was used to measure the protein concentration. Total $60 \mu \mathrm{g}$ of protein were separated on $10 \%$ SDS-PAGE gels and blotted onto nitrocellulose membranes. The membranes were blocked for 2 hours with 5\% non fat dry milk diluted with tris buffered saline (TBS) and incubated with primary antibodies overnight at $4^{\circ} \mathrm{C}$. The membranes were washed with TBST, and then incubated with appropriate horseradish peroxidase-conjugated secondary antibody for $1 \mathrm{~h}$ at $37^{\circ} \mathrm{C}$. Enhanced chemiluminescence reagent (Merck Millipore, Germany) was used to detect the signal on the membrane. Western blot bands were quantified by the mean gray value using NIH Image J 7.0. The expression of genes was analyzed by normalizing to the expression of the internal control (GAPDH).

\section{ELISA}

ELISA kits were used to detect the levels of ACE and s-Flt in the serum according to manufacturer's instructions.

\section{Statistical analysis}

Statistical analyses were performed using Graphpad Prism 5 software (Graphpad Software, Inc., La Jolla, CA, USA) and the data are presented as the mean \pm standard deviation. An unpaired two tailed Student's test or one way analysis of variance (ANOVA) with Bonferroni $t$ post-test was used to analyze the data depending on conditions. $\mathrm{P}<0.05$ was considered to indicate a statistically significant difference.

\section{Author contributions}

YG, YLF, JJY conceived of the study, and participated in its design and coordination and helped 
to draft the manuscript. HSC, YXY and HY participated in the design of the study and performed the statistical analysis. J D, JZ, YHX, JJX carried out the animal experiments. YG, YLF, JZ, YHX, JJX carried out molecular experiments and participated in data analysis and interpretation. All authors read and approved the final manuscript.

\section{CONFLICTS OF INTEREST}

No conflict of interest exits in this study.

\section{FUNDING}

This work was supported by Jiangsu provincial Science and Technology Department of the key disease standardized diagnosis and treatment project (No. BE2015618); standardized diagnosis and treatment projects research program of Medical Science and Technology Development Fund of the Medical Control Center in Wuxi City (No. YGZXG1408); Nature Sciences Foundation of Jiangsu Province (No. BK20141100); Wuxi Maternal and Child Health-Care Hospital Dr. start fund research projects (No. 2014BS06); and Maternal and Child Health research project in Jiangsu province (F201422).

\section{REFERENCES}

1. Phipps E, Prasanna D, Brima W, Jim B. Preeclampsia: updates in pathogenesis, definitions, and guidelines. Clin J Am Soc Nephrol. 2016; 11:1102-1113.

2. Zhou X, Niu JM, Ji WJ, Zhang Z, Wang PP, Ling XF, Li YM. Precision test for precision medicine: opportunities, challenges and perspectives regarding pre-eclampsia as an intervention window for future cardiovascular disease. Am J Transl Res. 2016; 8:1920-1934.

3. Hansson SR, Naav A, Erlandsson L. Oxidative stress in preeclampsia and the role of free fetal hemoglobin. Front Physiol. 2014; 5:516.

4. Camarena PE, Garcia BL, Panduro BJ, Pascoe GS, Madrigal SA, Garcia PF, Totsuka SS. Efficacy of L-arginine for preventing preeclampsia in high-risk pregnancies: a doubleblind, randomized, clinical trial. Hypertens Pregnancy. 2016; 35:217-225.

5. Hung TH, Skepper JN, Burton GJ. In vitro ischemiareperfusion injury in term human placenta as a model for oxidative stress in pathological pregnancies. Am J Pathol. 2001; 159:1031-1043.

6. Klein E, Schlembach D, Ramoni A, Langer E, Bahlmann F, Grill S, Schaffenrath H, van der Does R, Messinger D, Verhagen-Kamerbeek WD, Reim M, Hund M, Stepan H. Influence of the sFlt-1/P1GF ratio on clinical decisionmaking in women with suspected preeclampsia. PLoS One. 2016; 11:e156013.
7. Liu X, Deng Q, Luo X, Chen Y, Shan N, Qi H. Oxidative stress-induced Gadd45alpha inhibits trophoblast invasion and increases sFlt1/sEng secretions via p38 MAPK involving in the pathology of pre-eclampsia. J Matern Fetal Neonatal Med. 2016; 29:3776-3785.

8. Feng Y, Hu L, Xu Q, Yuan H, Ba L, He Y, Che H. Cytoprotective role of alpha-1 antitrypsin in vascular endothelial cell under hypoxia/reoxygenation condition. J Cardiovasc Pharmacol. 2015; 66:96-107.

9. Feng Y, Xu J, Zhou Q, Wang R, Liu N, Wu Y, Yuan H, Che H. Alpha-1 antitrypsin prevents the development of preeclampsia through suppression of oxidative stress. Front Physiol. 2016; 7:176.

10. Wojciak-Stothard B, Tsang LY, Haworth SG. Rac and Rho play opposing roles in the regulation of hypoxia/ reoxygenation-induced permeability changes in pulmonary artery endothelial cells. Am J Physiol Lung Cell Mol Physiol. 2005; 288:L749-L760.

11. Zhou L, Qiao F. Expression of RhoA in placenta of preeclampsia. J Huazhong Univ Sci Technolog Med Sci. 2006; 26:744-746.

12. Mishra N, Nugent WH, Mahavadi S, Walsh SW. Mechanisms of enhanced vascular reactivity in preeclampsia. Hypertension. 2011; 58:867-873.

13. Shi J, Wei L. Rho kinases in cardiovascular physiology and pathophysiology: the effect of fasudil. J Cardiovasc Pharmacol. 2013; 62:341-354.

14. Butruille L, Mayeur S, Duparc T, Knauf C, Moitrot E, Fajardy I, Valet P, Storme L, Deruelle P, Lesage J. Prenatal fasudil exposure alleviates fetal growth but programs hyperphagia and overweight in the adult male rat. Eur $\mathbf{J}$ Pharmacol. 2012; 689:278-284.

15. Jiang ZH, Zhang TT, Zhang JF. Protective effects of fasudil hydrochloride post-conditioning on acute myocardial ischemia/reperfusion injury in rats. Cardiol J. 2013; 20:197-202.

16. Niermann C, Gorressen S, Klier M, Gowert NS, Billuart P, Kelm M, Merx MW, Elvers M. Oligophrenin1 protects mice against myocardial ischemia and reperfusion injury by modulating inflammation and myocardial apoptosis. Cell Signal. 2016; 28:967-978.

17. Luo SY, Chen S, Qin YD, Chen ZW. Urotensin-receptor antagonist SB-710411 protects rat heart against ischemiareperfusion injury via RhoA/ROCK pathway. PLoS One. 2016; 11:e146094.

18. Kiss A, Tratsiakovich Y, Gonon AT, Fedotovskaya O, Lanner JT, Andersson DC, Yang J, Pernow J. The role of arginase and rho kinase in cardioprotection from remote ischemic perconditioning in non-diabetic and diabetic rat in vivo. PLoS One. 2014; 9:e104731.

19. Liu H, Chen X, Han Y, Li C, Chen P, Su S, Zhang Y, Pan Z. Rho kinase inhibition by fasudil suppresses lipopolysaccharide-induced apoptosis of rat pulmonary 
microvascular endothelial cells via JNK and p38 MAPK pathway. Biomed Pharmacother. 2014; 68:267-275.

20. Wang QM, Stalker TJ, Gong Y, Rikitake Y, Scalia R, Liao JK. Inhibition of Rho-kinase attenuates endothelialleukocyte interaction during ischemia-reperfusion injury. Vasc Med. 2012; 17:379-385.

21. Tsounapi P, Saito M, Kitatani K, Dimitriadis F, Ohmasa F, Shimizu S, Kinoshita Y, Takenaka A, Satoh K. Fasudil improves the endothelial dysfunction in the aorta of spontaneously hypertensive rats. Eur J Pharmacol. 2012; 691:182-189.

22. Gibson CL, Srivastava K, Sprigg N, Bath PM, Bayraktutan U. Inhibition of Rho-kinase protects cerebral barrier from ischaemia-evoked injury through modulations of endothelial cell oxidative stress and tight junctions. J Neurochem. 2014; 129:816-826.

23. Kajimoto H, Hashimoto K, Bonnet SN, Haromy A, Harry G, Moudgil R, Nakanishi T, Rebeyka I, Thebaud B, Michelakis ED, Archer SL. Oxygen activates the Rho/Rhokinase pathway and induces RhoB and ROCK-1 expression in human and rabbit ductus arteriosus by increasing mitochondria-derived reactive oxygen species: a newly recognized mechanism for sustaining ductal constriction. Circulation. 2007; 115:1777-1788.

24. Antoniu SA. Targeting RhoA/ROCK pathway in pulmonary arterial hypertension. Expert Opin Ther Targets. 2012; 16:355-363.

25. Surma M, Wei L, Shi J. Rho kinase as a therapeutic target in cardiovascular disease. Future Cardiol. 2011; 7:657-671.

26. Klopocka W, Redowicz MJ. Rho/Rho-dependent kinase affects locomotion and actin-myosin II activity of Amoeba proteus. Protoplasma. 2004; 224:113-121.

27. Minambres R, Guasch RM, Perez-Arago A, Guerri C. The RhoA/ROCK-I/MLC pathway is involved in the ethanol-induced apoptosis by anoikis in astrocytes. J Cell Sci. 2006; 119:271-282.

28. Song Y, Hoang BQ, Chang DD. ROCK-II-induced membrane blebbing and chromatin condensation require actin cytoskeleton. Exp Cell Res. 2002; 278:45-52.

29. Nunes KP, Rigsby CS, Webb RC. RhoA/Rho-kinase and vascular diseases: what is the link? Cell Mol Life Sci. 2010; 67:3823-3836.

30. Rankinen $\mathrm{T}$, Church $\mathrm{T}$, Rice $\mathrm{T}$, Markward N, Blair $\mathrm{SN}$, Bouchard C. A major haplotype block at the rhoassociated kinase 2 locus is associated with a lower risk of hypertension in a recessive manner: the HYPGENE study. Hypertens Res. 2008; 31:1651-1657.

31. Seasholtz TM, Wessel J, Rao F, Rana BK, Khandrika S, Kennedy BP, Lillie EO, Ziegler MG, Smith DW, Schork NJ, Brown JH, O'Connor DT. Rho kinase polymorphism influences blood pressure and systemic vascular resistance in human twins: role of heredity. Hypertension. 2006; 47:937-947.

32. Li H, Peng W, Jian W, Li Y, Li Q, Li W, Xu Y. ROCK inhibitor fasudil attenuated high glucose-induced MCP-1 and VCAM-1 expression and monocyte-endothelial cell adhesion. Cardivasc Diabetol. 2012; 11:65.

33. Santen S, Wang Y, Laschke MW, Menger MD, Jeppsson $\mathrm{B}$, Thorlacius $\mathrm{H}$. Rho-kinase signalling regulates CXC chemokine formation and leukocyte recruitment in colonic ischemia-reperfusion. Int J Colorectal Dis. 2010; 25:1063-1070.

34. Lopez NC, Ebensperger G, Herrera EA, Reyes RV, Calaf G, Cabello G, Moraga FA, Benaldo FA, Diaz M, Parer JT, Llanos AJ. Role of the RhoA/ROCK pathway in highaltitude associated neonatal pulmonary hypertension in lambs. Am J Physiol Regul Integr Comp Physiol. 2016; 310:R1053-R1063. 